\title{
What to expect when you learn to expect: temporal expectations as a perceptual learning process
}

Orit Shdeour $^{1}$, Noam Tal-Perry ${ }^{1}$, Moshe Glickman ${ }^{2,3} \&$ Shlomit Yuval-Greenberg ${ }^{1,4}$

\author{
${ }^{1}$ School of Psychological Sciences, Tel-Aviv University, Israel \\ ${ }^{2}$ Department of Experimental Psychology, University College London, United \\ Kingdom \\ ${ }^{3}$ Max Planck Centre for Computational Psychiatry and Ageing Research, University \\ College London, United Kingdom \\ ${ }^{4}$ Sagol School of Neuroscience, Tel-Aviv University, Israel
}

Orit Shdeour (iD https://orcid.org/0000-0002-2858-113X

Noam Tal-Perry (iD https://orcid.org/0000-0003-2521-9546

Moshe Glickman (D) https://orcid.org/0000-0002-3792-1992

Shlomit Yuval-Greenberg (D) https://orcid.org/0000-0001-6455-7578

Correspondence concerning this article should be addressed to Orit Shdeour, School of Psychological Sciences, Tel-Aviv University, P.O. box 39040, Tel Aviv 6997801. Email: oritshdeour@mail.tau.ac.il 


\begin{abstract}
The formation of temporal expectations depends on prior experience with temporal regularities over-time, but its link with perceptual learning processes has never been investigated. In this study, we employed a novel approach for studying temporal expectations as a learning process, by examining how prior experience with certain temporal dynamics affects the ability to form new expectations. Our findings reveal that participants, who were initially exposed to a fixed temporal routine, showed a performance decline and a reduced ability to adapt when this routine was changed. In contrast, participants who were initially presented with a random routine showed higher performance and quick adaptation following a change. Corroborated with eye-tracking and computational modeling, these findings suggest that forming temporal expectations is based on learning of a specific temporal rule which cannot be easily unlearned or generalized. This is first-time evidence that the formation of temporal expectation follows the principles of perceptual learning.
\end{abstract}

\title{
Introduction
}

Sitting in the driver-seat waiting for the light to change to green, holding up a hand waiting for a teacher to call on us, or standing at the bus-stop waiting for the bus to arrive; our daily lives are full of scenarios in which temporal expectations shape our behavior ${ }^{1}$. Forming new temporal expectations requires extensive prior experience with specific temporal regularities and depends on repeated exposures to the same timing routine $^{2}$. For example, our ability to anticipate the exact moment when a streetlight would switch from yellow to green depends on our numerous previous encounters with traffic lights of the same timing routine. Expectations regarding the timing of the green light are likely to enhances our perception and improve our ability to react to it by depressing the gas pedal quickly once it appears. However, the underlying mechanisms by which temporal expectations are created and formed, and the way they affect latter ability to construct new regularities is still unknown. 
Decades of research on perceptual learning have thoroughly examined how experience with stimuli and tasks shapes perception, attention, and behavior ${ }^{3-7}$. These studies have consistently shown that, following practice, there is a typical sustainable improvement in performance on perceptual tasks. However, despite its robustness, this improvement is often very specific - performance improves on the trained task with the trained set of stimuli, but rarely on other, even similar, tasks or stimuli ${ }^{5,8,9}$. This extensive knowledge on perceptual learning processes and their specificity could be useful when aiming to understand how temporal expectations are formed based on prior experiences. Studying temporal expectation as a perceptual learning process could shed light on the mechanisms by which temporal expectations are formed and on how new expectations evolve from existing ones. Surprisingly, however, the link between these perceptual learning and temporal expectation processes has never been investigated.

Perceptual learning typically involves explicit training of the acquired perceptual skill. For example, studies on perceptual learning in the time domain focused on the effects of training on explicit timing tasks, such as interval discrimination ${ }^{10,11}$ and reproduction ${ }^{12}$. In contrast, the formation of temporal expectations depends on implicit learning of temporal regularities and relies on exposure to repeating temporal information. Understanding the role of implicit learning of environmental regularities, is crucial for explaining how expectations are formed, maintained, and updated.

In this pre-registered study, we have investigated how implicit acquisition of one temporal regularity affects the ability to construct expectations based on a new one. The goal of this study was to contrast two alternative hypotheses: (a) that implicit learning of one temporal regularity is non-specific learning of strategy as it enhances the formation of temporal expectations based on other regularities; and (b) that learning temporal regularities is specific and therefore hinders the learning of new ones and 
reduced the ability to form new expectations. The behavioral findings, corroborated with eye-tracking and computational modeling, provided strong support for the second hypothesis. The findings reveal that participants who had prior experience with one temporal regularity showed a sharp decline in performance following the unannounced introduction of a new regularity and were slower to adapt to it, relative to participants who had prior experience with a random timing routine. This indicates that, akin to other perceptual learning mechanisms, the formation of temporal expectations is specific to the learned temporal regularity. This first-time evidence supports the conceptualization of temporal expectation as a perceptual learning process

The finding that learning in temporally random environment enhances flexibility and promotes the ability to adapt and construct new expectations, has broad implications that go well beyond research of temporal expectations. Studies of human and non-human learning systems has examined the effect of noise on the generalizability of these systems. Recent machine learning research has shown that adding noise to trained exemplars may improve the generalizability of learning algorithms as it reduces the risk of overfitting ${ }^{13}$. Findings of this study demonstrate equivalent results in humans: exposure to a temporally-noisy conditions can promote adaptation to new environments and improve perceptual performance. 
(A)

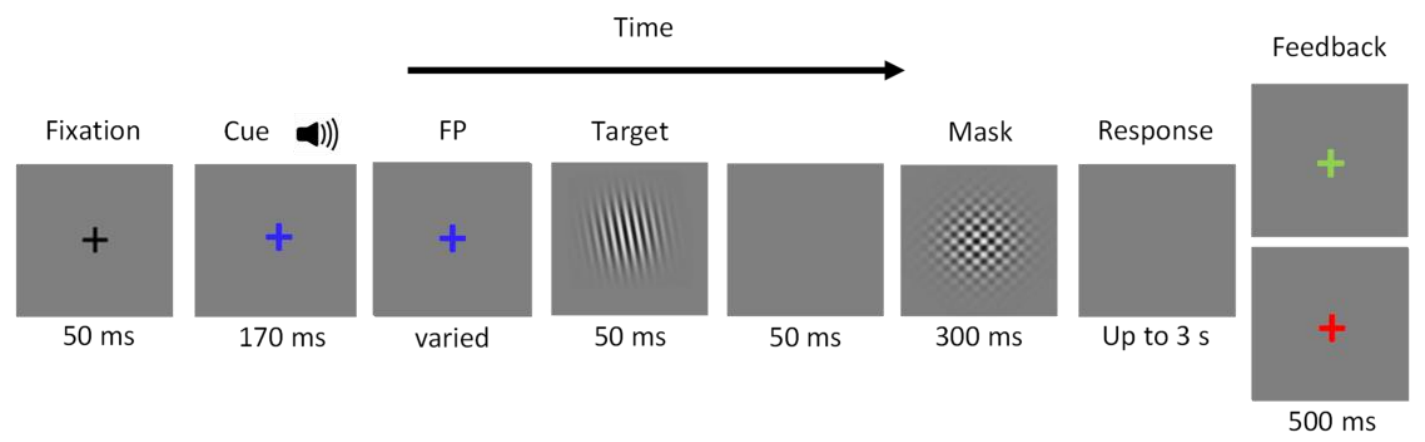

(B)

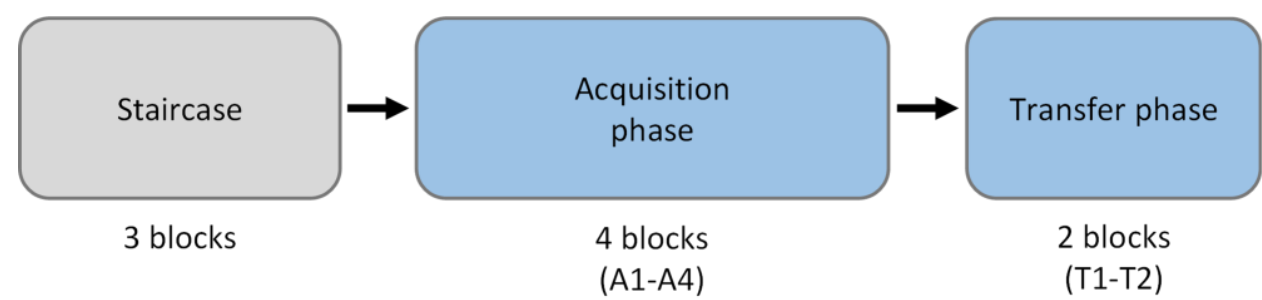

Fig 1. Procedure. (A) Trial procedure. Every trial began with a black fixation cross followed by a simultaneous presentation of auditory (pure tone) and visual (blue fixation cross) cues. The blue fixation cross continued to be presented during the interval between cue onset and the target onset (foreperiod, FP). The duration of the foreperiod varied according to the phase and group conditions. After the foreperiod, the target was presented and was followed by a mask. Following target presentation, participants were asked to determine whether the target was tilted left or right and respond by pressing one of two buttons, and then received feedback (green or red fixation cross for correct or incorrect responses, respectively). After the feedback, there was a variable interval of 0.2$0.7 \mathrm{~s}$ before the next trial was initiated. (B) Experimental blocks. The experiment included a total of seven blocks: In three preliminary staircase blocks, the tilt threshold was chosen individually per participant to achieve approximately $80 \%$ accuracy rates. This was followed by the main experiment that included four acquisition blocks, and two transfer blocks. Each experimental block included 96 trials.

\section{Results}

In this study we have employed a common experimental paradigm, the foreperiod paradigm, which creates an association between a warning signal (cue) and a target, with a fixed or varying interval between them, called a foreperiod $(\mathrm{FP})^{14-20}$. It was repeatedly shown that when the foreperiods are fixed and targets are rendered predictable, behavioral performance is enhanced, relative to when foreperiods are variable and targets are unpredictable ${ }^{21,22}$. Two groups of participants performed the foreperiod paradigm with a visual discrimination task

(Fig 1A). In the first phase of the experiment (acquisition phase), participants of one group 
(fixed group) were given the chance to construct temporal expectations, as they were exposed solely to fixed foreperiods of $2700 \mathrm{~ms}$. Participants of a second group (random group) were not given this chance as they were exposed to random foreperiods (of 1700-3700 ms), changing from one trial to the next. In the second phase of the experiment (transfer phase), both groups performed the same task with a mostly-fixed foreperiod of $700 \mathrm{~ms}$ (Fig 1B).

\section{Accuracy rates}

Accuracy results were analyzed by constructing a three-way GLM model assuming a binomial family of response and a logit link. The model includes the fixed factors Trial Number (modeled with 1st polynomial, i.e., linear component), Group (fixed/ random) Phase (acquisition/ transfer) and their accompanying interaction terms, allowing for full random effect structure (random intercept, random slopes for each variable and their interactions, and accompanying correlation parameters).

This GLMM analyses revealed no main effect for Group $\left(\chi^{2}(1)=0.348, p=\right.$ $.556)$ nor for Trial number $\left(\chi^{2}(1)=0.642, p=.423\right)$, and the two factors did not significantly interact with each other $\left(\chi^{2}(1)=0.061, p=.805\right)$. However, there was a significant main effect for Phase $\left(\chi^{2}(1)=24.393, p<.0001\right)$, with an overall decrease in accuracy at the transfer phase $(\mathrm{M}=70.8 \%, \mathrm{SD}=12.0 \%)$ relative to the acquisition phase $(\mathrm{M}=80.4 \%, \mathrm{SD}=7.2 \%)$, indicating that the change in the foreperiod distribution from the acquisition to the transfer phase interfered with performance. The phase effect was significantly modulated by Trial number $\left(\chi^{2}(1)=8.104, p=\right.$ .004). This interaction resulted from a shallow decrease of accuracy over trials during the acquisition phase (log estimate $=-.077$, Wald's $\mathrm{z}=-2.042, p=.041)$, possibly due to fatigue, and an overall increase in accuracy during the transfer phase (log estimate $=.177$, Wald's $\mathrm{z}=3.278, p=.001$ ), possibly due to the gradual adaptation for the new foreperiod routine. 
Critical for the purpose of this study is the significant two-way interaction between Group and Phase $\left(\chi^{2}(1)=8.149, p=.004\right)$. A follow-up analysis performed separately on the two phases revealed that accuracy rates for the two groups were similar in the acquisition phase (log estimate $=-.105$, Wald's $\mathrm{z}=-.743, \mathrm{p}=.458)$, but differed significantly in the transfer phase (log estimate $=.462$, Wald's $z=2.473, p=$ .0134). The random group showed higher accuracy in the transfer phase ( $M=74.9 \%$, $\mathrm{SD}=12.7 \%)$ than the fixed group $(\mathrm{M}=66.9 \%, \mathrm{SD}=10.1 \%$; Fig 2a), consistent with our hypothesis that exposure to random intervals improves adaptation relative to fixed intervals.

Furthermore, we observed a significant three-way interaction between Phase, Group, and Trial number $\left(\chi^{2}(1)=4.907, p=.027\right)$, indicating that the modulation of Group by Phase evolved over trials. Again, we performed a follow-up analysis separately on the two phases. In the acquisition phase, both groups showed a similar pattern of performance over trials, including a gradual decrease in performance (random group: $\log$ estimate $=-.055$, Wald's $\mathrm{z}=-1.043, p=.297$; fixed group: $\log$ estimate $=-.099$, Wald's $z=-1.845, p=.065)$, which was likely due to fatigue. In contrast, during the transfer phase (see Fig. 2b), the groups differed in their performance dynamics over trials. Whereas at the beginning of the transfer phase, both groups showed a sharp decline in performance relative to the acquisition phase (see Fig. 2a); the fixed group remained at a low and stable performance level throughout the phase (log estimate $<-0.001$, Wald's $z=-0.005, p=.99)$ while the random group quickly recovered and showed an overall gradual enhancement over trials (log estimate $=0.200$, Wald's $z=2.587, p=.0097)$. These findings support the 
hypothesis that learning a systematic interval routine hinders participants' ability to adapt to new routines.

(A)

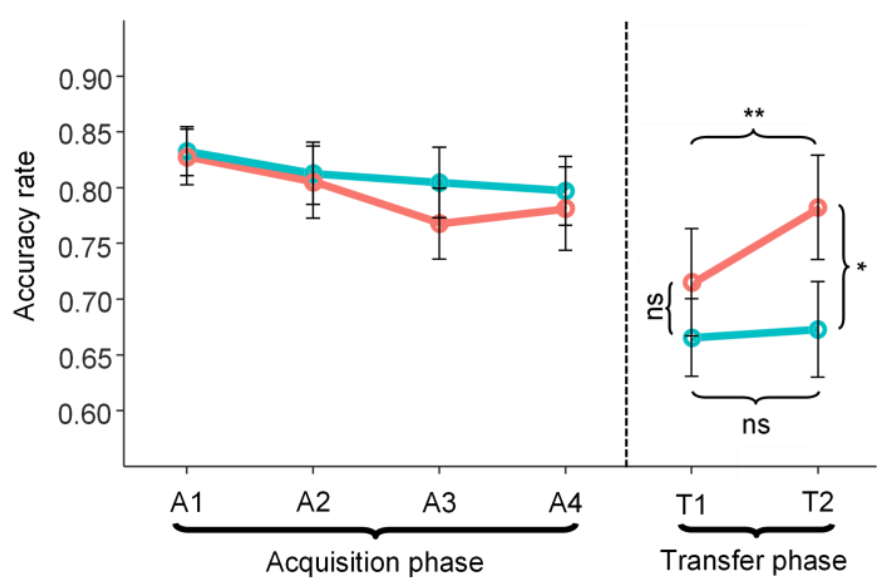

(B)

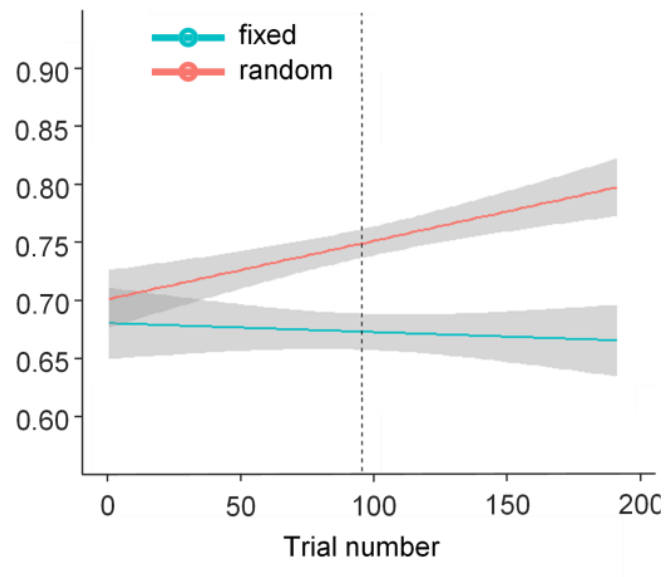

Fig 2. Accuracy rate results. (A) The $\mathrm{X}$-axis represents the experimental blocks in chronological order (Acquisition= A 1-4, Transfer $=$ T 1-2). Error bars depict \pm 1 standard error from the group mean. Simple effects are marked as: $\mathrm{p}<0.05^{*}, \mathrm{p}<0.01^{* *}$, ns=not significant. (B) Regression line of trial-wise temporal dynamic for accuracy rates in each group during the transfer phase, based on the individual data of the participants for each trial. Shaded area represents $95 \%$ confidence interval around the slope. Dashed line represents the division between blocks (T1 and T2).

\section{Speed-accuracy tradeoff}

In this study we used a difficult, non-speeded, task that is more suitable for studying accuracy rates and less for studying RTs. We, nevertheless, examined RTs to ensure that the observed accuracy-rate findings cannot be explained solely as speed-accuracy tradeoffs ${ }^{23-25}$. To this end, we inserted the RT results as an additional fixed factor in the accuracy model described above, along with its interactions with the other factors. We used this new model to examine whether our main accuracy finding - a significant three-way interaction of Phase, Group, and Trial number - can be explained by RTs or by its interactions with the other factors. Indeed, a significant three-way interaction of Phase X Group X Trial number was found with this new model $\left(\chi^{2}(1)=4.619, p=\right.$ $.032)$. 
A follow-up examination, performed separately on accuracy rates of the two phases revealed no evidence for a trend over-time in the fixed group in the acquisition phase (log estimate $=-.078$, Wald's $z=-1.554, p=.120)$ and only a marginal trend of decreasing accuracy over time in the random group during that phase (log estimate $=$ .090 , Wald's $z=-1.749, p=.080)$. However, in the transfer phase, we found a significant positive trend in the accuracy rates of the random group (log estimate $=$ .174 , Wald's $z=2.415, p=.016$ ), i.e., participants of this group improved their performance over time during the transfer phase; but there was no evidence for a trend in the fixed group (log estimate $=-.027$, Wald's $z=-.407, p=.684)$. These trends are consistent with our main findings, suggesting that this effect is independent of RT and cannot be explained by a speed-accuracy tradeoff. This conclusion is also supported by the result of a drift-diffusion model (DDM, described below), which takes both RT and accuracy rates into account. Following our pre-registered plan, we have also performed a full GLM of RT, which is provided in Supplementary information S2.

\section{Eye movements}

In a series of previous studies, we showed that mean saccade-rate at -100 to 0 relative to target onset (pre-target SR), can be used as an index for temporal expectation: pretarget SR was consistently found to be lower for expected relative to unexpected targets. Here, we employed a 2X2 mixed ANOVA with between-subject factor of Group (fixed /random) and within subject factor of Block (first/ second) on the pretarget SR in the transfer phase. We found no significant main effect for Group $(F(1$, $35)=1.543, p=.222)$ nor for Trial bin $(F(1,35)=3.366, p=.075)$, but there was a significant interaction between them $(F(1,35)=4.580, p=.039)$, indicating that pretarget SR was decreased over time in the random group, while it remained constant or even slightly increased in the fixed group (Fig 3). Follow up analysis demonstrated 
that at the beginning of the transfer phase (the first block), there was no significant difference between the groups $(t(35)=0.219, p=.828)$, but in at the end of the transfer phase (the second block), pre-target SR was higher for the fixed relative to the random groups $(t(35)=2.202, p=.034)$. When examining the effect of time on the two groups separately we found that the pre-target SR of the random group decreased dramatically between the early and the late trial bins $(t(18)=3.385, p=.003)$, whereas that of the fixed group has not changed significantly $(t(19)=0.192, p=.850)$.

(A)

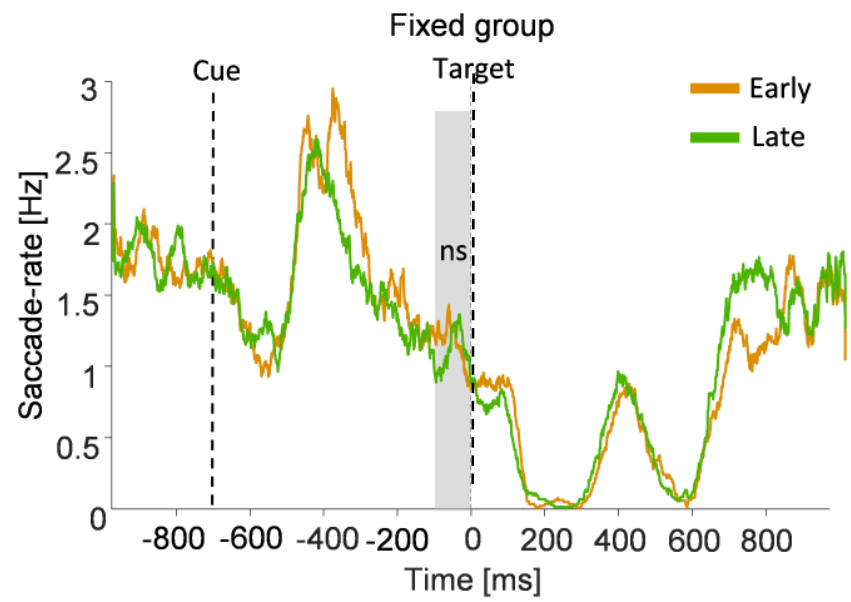

(B)

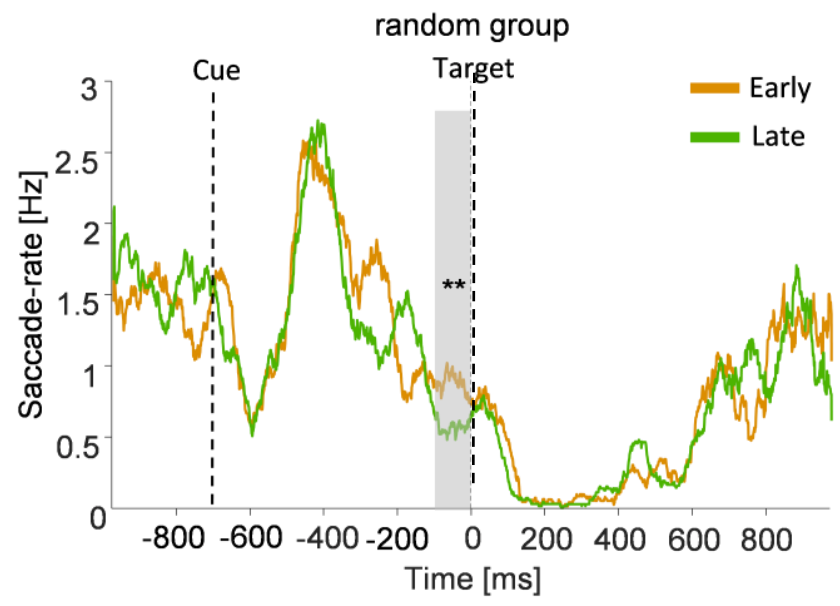

(C)

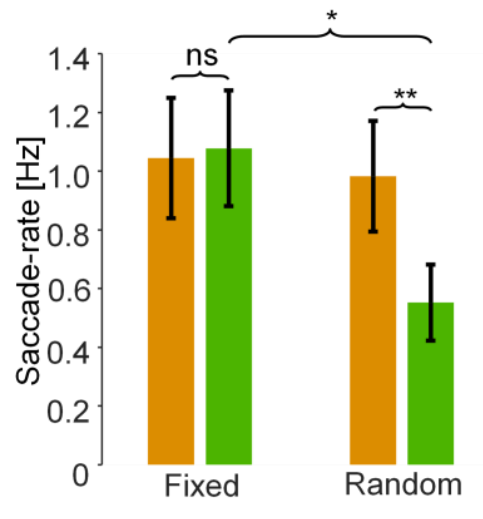

Fig 3. Saccade-rate in the transfer phase. Mean saccade-rate for the early (orange) and late (green) trial bins in the transfer phase, separately for the fixed (A) and the random (B) groups. Shadowed area represents the analyzed duration (-100 to $0 \mathrm{~ms}$, pre-target SR). The dashed lines represent the cue and target onsets. (C) Early and late pre-target SR in each group during the transfer phase. Significance of effects are marked as: $p<0.05^{*}, p<0.01^{* *}$ or ns (non-significant). 
In light of the previous findings ${ }^{14,15,18,26-28}$ who showed that a decrease in pretarget SR reflects enhanced temporal expectation, the present SR results complement the accuracy-rate findings and suggest that experiencing with fixed vs. random intervals, affects the pattern of adjustment to a new regularity.

In addition, we compared the pre-target SR of the groups during the acquisition phase using an independent samples t-test and found a significant difference between the groups $(t(37)=-2.081, \mathrm{p}=.044 ;$ Fig. 4): pre-target SR was higher for the random relative to the fixed group. This effect is consistent with the higher pre-target SR found in a random relative to a fixed condition in previous within-subject studies $15,27,28$ and is a first-time demonstration of this effect between groups.

(A)

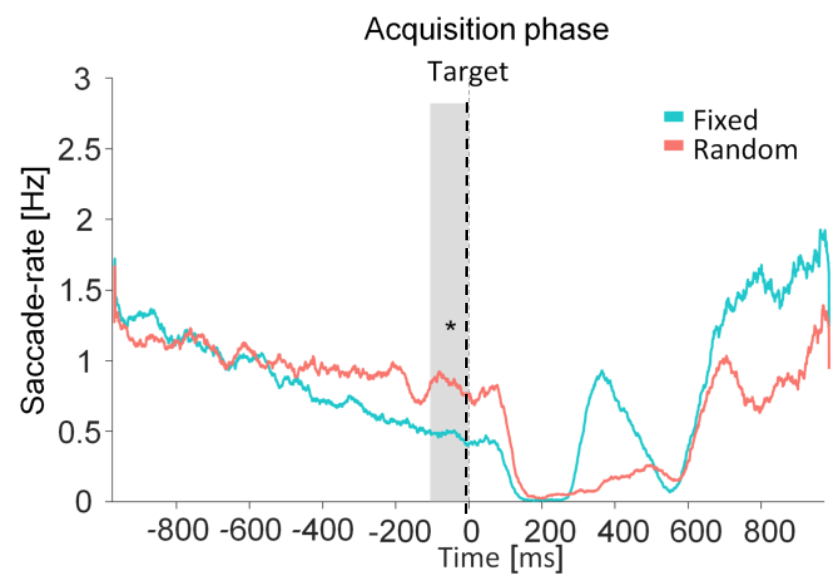

(B)

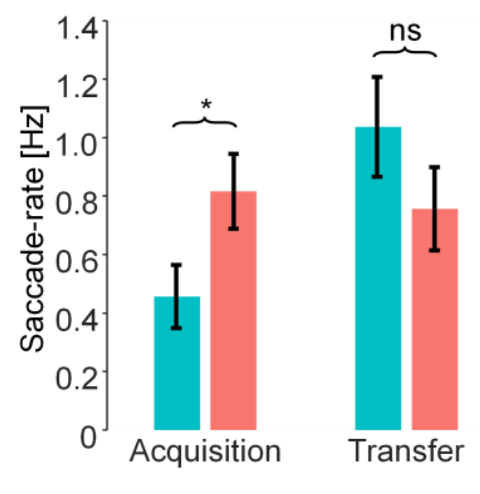

Fig 4. Saccade-rate in the acquisition phase. (A) Mean saccade-rate (SR) for the fixed (blue) and random (red) groups across time in the acquisition phase. Shadowed area represents the analyzed duration (-100 to $0 \mathrm{~ms}$, pre-target SR). The dashed line represents the target onset. Cue onset is outside the displayed time window. (B) Pre-target SR in both phases and groups. Error bars represent \pm 1 standard error from the group mean. The significant effect within the acquisition phase is marked: $\mathrm{p}<0.05^{*}, \mathrm{~ns}=$ not significant.

\section{Computational modeling: drift-diffusion model}

The Drift-Diffusion Model (DDM) ${ }^{29-31}$ was used to decompose task performance measures into interpretable psychological processes, in order to better identify the 
mechanisms underlying the effects obtained for accuracy and response times. The DDM assumes that evidence is integrated across time until reaching an internal decision-boundary, then a decision is triggered ${ }^{32-34}$. Here, we compared two variants of the DDM: the first model (hereafter phase-constant model) was a simple DDM with three free parameters: i) Drift-rate (v), indicating the signal-to-noise ratio of the evidence, ii) Boundary-separation (a), indicating the evidence required to make a response, and iii) Non-decision time ( $t 0)$, indicating the time of the processes that are not involved in the evidence accumulation (e.g., response execution). The second model (hereafter phase-varying model) was similar to the first model, except that these three parameters were allowed to vary across the acquisition and transfer phases (resulting in six free parameters). Note that, since the drift rate, boundary separation, and non-decision time were estimated separately for the acquisition and transfer phases, the phase-varying model can capture differences in the processing of the sensory stimuli between the acquisition and transfer phases. The models were fitted to the data of each subject using the Fast-DM 30.2 program $^{35}$.

The models were compared using the Bayesian Inference Criterion, which implements a trade-off between model goodness of fit and complexity by penalizing additional free parameters $(\mathrm{BIC})^{36}$. Model comparison showed that the phase-varying model decisively outperformed the phase-constant model $\left(\triangle \mathrm{BIC} \mathrm{C}_{\text {phase-constant }- \text { phase- }}\right.$ varying $=836$; values higher than 10 are considered strong evidence), indicating that phase significantly affects the cognitive process underlying the decision. Furthermore, to validate the phase-varying model, we simulated this model using the best-fitted parameters of the participants. The simulated data well captured the decrease in accuracy in the transfer-phase, and in particular the larger decrease shown by the 
fixed group (Fig. 5A). In addition, the phase-varying model well captured the RT patterns of the actual participants in both groups and phases (Fig. 5B).

Finally, we compared the best-fitted parameters of the phase-varying model obtained in the acquisition and transfer phases, separately for the participants in the fixed and random groups (see Supplementary information S3 for parameter recovery). We found that, for participants of the fixed group, the drift rate (indicating signal-tonoise ratio) was significantly higher at the acquisition stage than in the transfer phase $(t(19)=5.99, p<.001)$; but no such effect was found for the random group $(t(18)=$ $.98, p=.33)$. This indicates that the ability of the fixed group to process the target deteriorated once the temporal regularity changed at the onset of the transfer phase, whereas the ability of the random group to process the target remained unchanged. In addition, as a result of the staircase procedure, no difference between the drift rate of the fixed and random groups was found $(t(37)=1.47, p=.15)$ in the acquisition phase (indicating a similar signal-to-noise ratio). Most importantly, in the transfer phase, the drift rate of the fixed group was significantly lower than that of the random group $(t(37)=2.20, p=.03)$, indicating poorer ability to process the sensory input. No significant differences were found for the boundary parameters between the acquisition and transfer phases for both the fixed $(t(19)=.83, p=.42)$ and the random $(t(18)=1.62, p=.12)$ groups. The non-decision time was higher in the acquisition phase than in the transfer phase, for the fixed group $(t(19)=2.72, p=.01)$, and a marginally significant trend in the same direction was found for the random group $(t(18)=1.98, p=.06)$. This could possibly result from an improvement in the motor execution due to practice effect. 
(A)

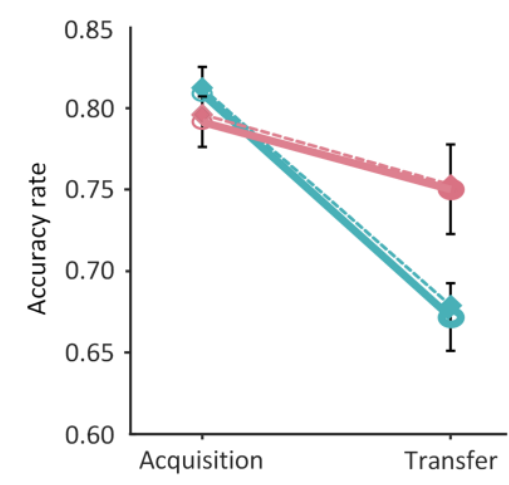

(C)

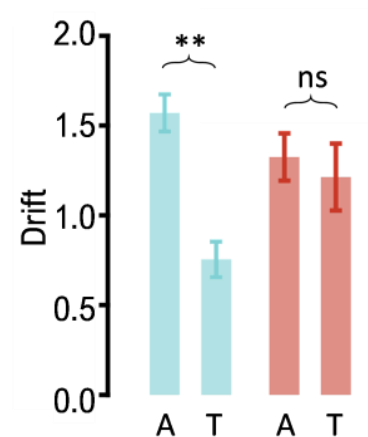

(D)

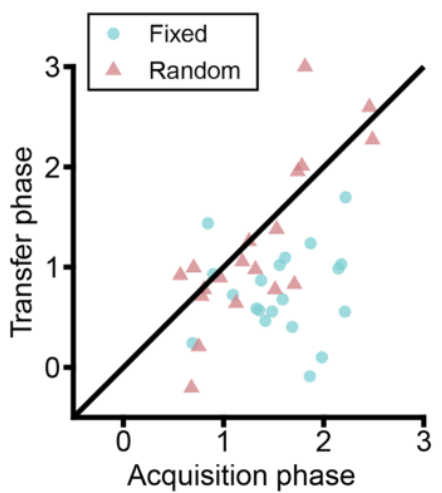

(E)

(F)
(B)

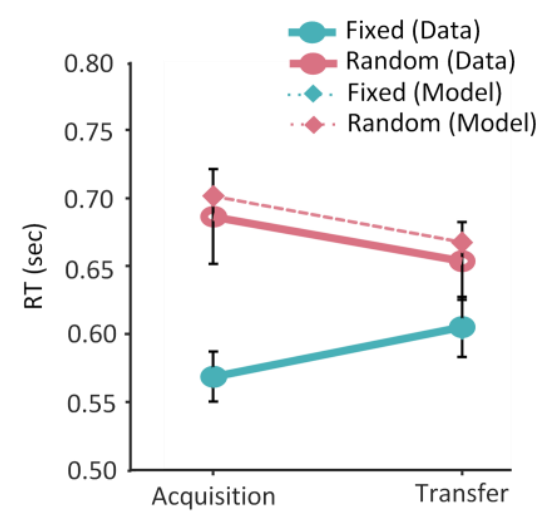

(G)

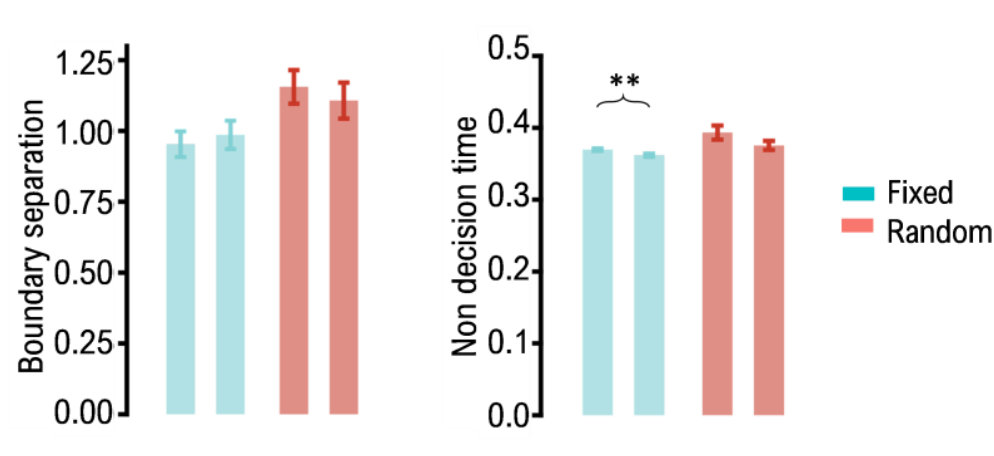

(H)
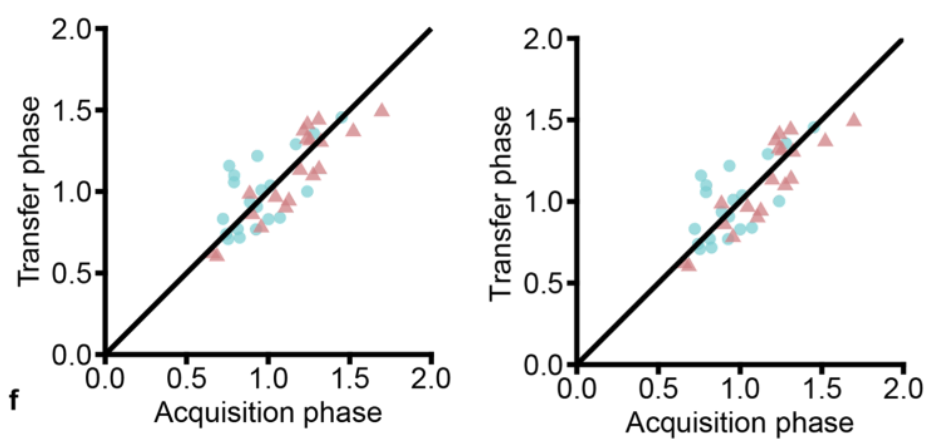

Fig 5. Drift Diffusion Model results. (A-B) Accuracy and RTs of the fixed (blue) and random (red) groups in the transfer and acquisition stages for both the real data (solid lines) and model predictions (dashed lines). Error bars are within-subjects SE. (C) Mean drift fit for the fixed (blue) and random (red) groups for the acquisition (A) and transfer (T) phases. Error bars represent \pm 1 standard deviation from the condition's mean; (D) Single-subject drift fit for the fixed (blue) and random (red) groups and each phase, with the diagonal line representing the identity line (drift fit in acquisition phase $=$ drift fit in transfer phase); (E-F) Mean and single-subject boundary separation fits; (G-H) Mean and single-subject null decision point fits. The significant effects between phases within group are marked: $\mathrm{p}<0.01^{* *}, \mathrm{~ns}=$ not significant. 


\section{Discussion}

In this study we examined how prior experiences with different temporal patterns affect adaptation to a new temporal regularity. Our findings show that prior exposure to a regular, relative to a variable temporal environment, hinders adaptation to a new regular environment and interferes with the formation of temporal expectations. Specifically, participants who were previously exposed to a random temporal pattern, adapted more effectively to a new fixed temporal pattern, than participants who were previously exposed to a fixed (but different) temporal pattern. While both groups of participants showed an initial decline in performance following the change in temporal pattern, the participants who were exposed to the random pattern recovered from this decline and showed an enhancement of performance between the first and the second blocks of the transfer phase, indicating that they have adjusted to the new time routine. In contrast, participants who were exposed to a fixed temporal pattern did not recover from this initial drop and their performance remained low until the end of the session, indicating that they have not adapted to the new regularity. A drift-diffusion model, fitted to the behavioral data (accuracy rates and reaction times), suggested that this effect is due to enhancement of sensory processing (i.e., higher signal-to-noise level) in the random group relative to the fixed group. These findings indicate that the implicit learning of temporal regularities is specific to the learned timing routine. Consequently, the formation of temporal expectation can be considered a specific perceptual learning process.

\section{Eye movements}

Eye-tracking provided further support for our hypothesis that the formation of temporal expectations is specific to the learned regularity. Previous studies revealed 
that when a target is predictable, there is a decrease in saccade rate (SR) shortly prior to the predicted target onset (pre-target SR), relative to when the target is unpredictable ${ }^{15,18}$. This saccade reduction, termed the pre-target saccadic inhibition, was found in various, even non-visual modalities ${ }^{14,26}$ and is thought to reflect anticipation processes ${ }^{27,28}$.

Here, we found that the pre-target SR of the random group decreased between blocks of the transfer phase, and this effect was not observed for the fixed group. Since previous studies suggested that reduction in saccade rate, i.e., enhancement of inhibition, reflects increasing temporal expectation, we propose that these findings reflect the evolvement of pre-target anticipation in the random group, but not in the fixed group. Consistently with the behavioral results, these findings suggest that prior experience with a fixed regularity hindered participants' ability to construct new temporal expectations based on a new regularity.

\section{Learning in contexts of variability}

Our findings reveal the superiority of learning in a temporally random condition relative to learning in a fixed one: prior exposure to temporal uncertainty enhanced adaptation to a new timing regularity, while learning a temporal system was found to be disadvantageous in a changing environment. Previous studies have demonstrated the importance of noise and uncertainty for learning processes in a variety of human and non-human learning systems. Machine learning studies demonstrated the central role of noise in non-human learning. These studies found that adding a certain amount of noise to a trained dataset, enhances performance of learning algorithms on untrained datasets ${ }^{37,38}$. This effect was offered as a solution to 
the "over-fitting problem", by which learning algorithm are trained to fit an existing environment, by fail to generalize when this environment changes ${ }^{13,39,40}$.

Our findings are also consistent with previous human studies of different fields of cognitive research, that have demonstrated how learning within a context of variability, uncertainty, or randomness, can be advantageous. Perceptual learning studies showed that when a few tasks or stimuli are interleaved during practice, learning can be transferred to untrained features or stimuli ${ }^{41-43}$. Moreover, it was shown that interleaved training on two perceptual tasks can improve the performance of both even if neither received sufficient training to promote performance on its own $\mathrm{n}^{44,45}$. Not only perceptual learning gains from uncertainty but also motor learning. The Contextual interference effect is a known phenomenon where interference during practice can be beneficial to skill learning. Studies of contextual interference in motor skill learning typically compared two groups who learn a set of motions either in a blocked practice schedule, i.e., by practicing a single motion throughout the block; or in a random practice schedule, i.e., by practicing a variety of randomly intermittent motions. Despite poorer performance in participants who practice the random relative to the blocked routines, this group of participants also showed enhanced long-term retention and transfer of the motor skills, demonstrating the beneficial effect of the random practice routine ${ }^{46,47}$. It was further suggested that, under random conditions, participants go through deeper and more elaborate processing of the skill or perceptual task that they practiced ${ }^{48}$, and this hypothesis was supported by fMRI findings showing higher activity in sensorimotor and premotor regions when participants practiced a random variety of motor skills relative to practicing only one skill at a time ${ }^{49}$. 
Our present findings are consistent with these studies and with the notion that an unstable or random environment is beneficial for learning. In our study, participants performed training of a perceptual task in one temporal context (i.e., a specific foreperiod distribution) and, were then tested in another context (i.e., a new foreperiod distribution). Consistent with the previous studies, we find that exposure to a varied temporal context promoted performance and transfer relative to training in a non-varied context.

\section{A Bayesian interpretation}

Thus far, we focused on the interpretation of our findings as reflecting the perceptual learning processes involved in the formation of temporal expectations. A different, but not mutually exclusive, interpretation of these findings is provided by the Bayesian approach. The Bayesian framework proposes that our experience of the world is shaped by the integration of bottom-up sensory input (likelihood) and top-down expectations, which are based on prior knowledge on the probabilistic distribution of the target ${ }^{50-54}$. According to this model, the balance between prior and likelihood is based on precision - we prefer to rely on information that is more precise, i.e., has a narrower distribution. Thus, when sensory information is ambiguous, we rely more on our prior knowledge, and when priors are inconsistent, we rely more on sensory evidence ${ }^{52,55,56}$.

In 'classical' Bayesian studies, priors typically consist of information that can be used directly and explicitly to support decisions regarding the target. However, here we examined temporal expectation, which is an implicit manifestation of temporal perception. Temporal expectation is assessed indirectly, by examining the effect of the temporal regularity on the target's perception. With such implicit 
measurements, priors are defined more loosely as any information that contributes to the target's perception but does not necessarily lead to it directly. The present findings could be interpreted as the result of an integration of such a "loose" temporal prior with the present temporal evidence.

In our experiment, the fixed group was presented with a very narrow distribution of foreperiods during the acquisition phase, while the random group was presented with a wider distribution. It could be hypothesized that, when integrating sensory information with prior knowledge of the foreperiod distribution, participants of the fixed group relied more on the prior (the acquisition phase distribution) than the random group. During the transfer phase, when encountered with a timing system that was new to both groups, the fixed group faced a large and interfering gap between the new system and their strong and highly-weighted prior. The random group also faced a gap, but it was smaller and less interfering than that of the fixed group, as their prior was not as highly weighted. During the transfer phase, they relied more on current sensory information than on the priors learned during the acquisition phase and, consequently, it was easier for them to flexibly adjust their predictions according to the new environment, than for the fixed group.

Therefore, our findings provide first-time evidence for the involvement of Bayesian processes in the field of temporal expectation. This is consistent with previous studies who showed that timing behavior was compatible with the prediction of the Bayesian model ${ }^{57-59}$, supporting the hypothesis that this model is fundamental to various aspects of human sensorimotor control and learning ${ }^{52}$.

This Bayesian interpretation of our findings is also consistent with findings of the drift-diffusion model. The higher drift-rate parameter which was attributed by the 
model to the random group in the transfer phase, suggests that sensory processing was higher in this group than in the fixed group, i.e., participants of the random group perceived the target at a higher signal-to-noise level ${ }^{60}$. This is consistent with the Bayesian interpretation suggesting that the random group relied more on the likelihood - current temporal regularity - and therefore experienced enhanced perceptual processing during the transfer phase.

\section{Conclusion}

Our findings support the hypothesis that, akin to explicit perceptual learning of temporal patterns, the construction of temporal expectations is specific and cannot be easily generalized. The findings show that exposure to one temporal routine results in the development of expectation to a specific set of temporal routines, that cannot be easily adjusted when the regularity changes. These findings contradict the intuitive notion that learning that the environment is systematic, would enhance the implicit learning of new rules. We conclude that, in the case of temporal regularities, learning that the world is systematic could interfere with adaptation to new systems, and may be counter-productive for perceptual performance. The finding that training in a temporally-unexpected environment is beneficial, has far-reaching implications for research on the effect of noise in human and computerized learning systems.

\section{Methods}

\section{Participants}

Participants included 40 students of Tel-Aviv University, divided in a double-blind manner into two groups of 20 participants each (Random group: 12 females, age: Mean 24.73, SD 3.71, Range 18-33; Fixed group: 11 females, age: Mean 25.7, SD 5.25, Range 19-43). One of the participants (random group) failed to perform the task 
during the acquisition phase, as indicated by mean accuracy of $58.85 \%$ during that phase, and was therefore excluded from analysis, based on the pre-registered exclusion criteria (link: https://osf.io/td5z3)

The sample size was determined based on a power analysis stimulation of pilot data of three participants in the fixed group and four participants in the random group. These data were not included in the main study (as mentioned in the pre-registration document; Power $=0.80, \alpha=0.05)$. All participants reported normal or corrected-tonormal vision and no history of neurological disorders. All were naïve to the purpose of this study. They participated in the experiment in exchange for course credit or monetary compensation. The ethical committees of Tel Aviv University and the School of Psychological Sciences approved the study. Before participation, all participants signed informed consent.

\section{Stimuli}

The stimuli included a black or blue fixation cross $\left(0.08 \times 0.08^{\circ}\right)$ on a mid-gray background. An auditory cue consisted of a pure tone of $5 \mathrm{KHz}$, played for $25 \mathrm{~ms}$. The target was a slightly tilted Gabor grating patch (visible diameter $3^{\circ}$, spatial frequency $5 \mathrm{cpd}$, contrast 0.7 ). The tilt degree was determined individually using an adaptive staircase procedure. The target stimulus was followed by a mask, composed of two overlaid Gabor grating patches (same parameters as the targets) in full contrast, tilted at 45 and 315 degrees.

\section{Procedure}

Setup. Participants were seated in a dimly lit sound-attenuated chamber, head resting on a chinrest at a distance of $100 \mathrm{~cm}$ from the display monitor (24" LCD ASUS VG248QE, 1,920 × 1,080 pixels resolution, $120 \mathrm{~Hz}$ refresh rate, mid-gray luminance 
was measured to be $110 \mathrm{~cd} / \mathrm{m}^{2}$ ). Auditory stimuli were administered through speakers located on both sides of the monitor. The experiment was generated and controlled using MATLAB R2015a (MathWorks Inc., Natick, MA, USA) with Psychophysics Toolbox v $3^{61}$.

Trial Procedure. Each trial began with the presentation of a central black fixation cross. Following $0.5 \mathrm{~s}$ of stable fixation $\left(<3^{\circ}\right.$ off center) as verified by a gaze contingent procedure and a random inter-trial interval (ITI) of 0.2-0.7 s (uniformly distributed), the fixation cross changed color to blue and, simultaneously, a pure tone was heard. Both the visual and the auditory signals served as concurrent temporal cues marking the onset of a foreperiod (FP). The blue fixation cross remained on the screen throughout the foreperiod interval and the auditory tone was paused after 25 ms. The reason for using both visual and auditory signals as cues was to increase the strength of the temporal information and thus enhance the formation of temporal expectation for the target stimuli ${ }^{62}$.

The foreperiod lasted for varying durations, depending on the group and the experimental phase (see below). Following the foreperiod, the target stimulus was briefly presented (for $50 \mathrm{~ms}$ ) at the center of the screen and after $50 \mathrm{~ms}$ of a blank screen, the backward mask was presented centrally for $300 \mathrm{~ms}$. Participants were asked to report the orientation of the target's tilt (clockwise / counterclockwise) by pressing one of two keys. When a response was detected or once $3 \mathrm{~s}$ had elapsed with no response, the fixation changed color again to green or red for $500 \mathrm{~ms}$ to provide feedback on task performance (correct or incorrect, respectively). A lack of response was considered as an incorrect response. Following the feedback, the next trial was initiated. The trial procedure is depicted in Fig. 1. 
Staircase procedure. A 1-up 3-down staircase procedure was performed on the tilt of the target stimulus during a separate session before the main experiment to determine individual thresholds. The purpose of the staircase session was to ensure that the task difficulty level of both groups would be similar and to avoid ceiling or floor effects. The starting tilt of the Gabor-patch was set at $10^{\circ}$ and participants performed three staircase blocks of five minutes each, using a staircase procedure, with logarithmic steps, which yielded accuracy rates of approximately $80 \%$. During the staircase procedure, foreperiods distribution was identical to the participant's acquisition phase, determined by their group. The average tilt of the last eight reversals was taken as the participant's tilt for the experimental blocks (both for the acquisition and the transfer phases). Following the pre-registered exclusion criteria, participants who failed to perform the task during the staircase procedure (performance at the chance level) or were assigned a tilt of more than $10^{\circ}$ following the staircase procedure were excluded from the experiment and did not proceed to the main experimental session. The resulting average tilt of the two groups was nearly identical (fixed group: $3.283^{\circ} \pm 2.252^{\circ} \mathrm{SD}$; random group: $3.135^{\circ} \pm 1.525^{\circ}$ ). Twentysix of the participants (divided equally between groups) did not perform any training in addition to the staircase procedure. However, while running the experiment we encountered some participants who were unable to perform the task, and therefore, we added 12 training trials before the staircase procedure, for the rest of the participants (14 participants). Two of the training trials were without a mask, to ensure that participants were familiar with the target and understood the task. This short training did not affect the tilt threshold or the performance during the acquisition or transfer phases. The full analysis of the staircase procedure can be found in Supplementary information S1. 
Experimental blocks. The main experimental session began with four blocks of the acquisition phase, followed by two blocks of the transfer phase (see Fig 1B), with each block consisting of 96 trials and breaks administered between them. During the acquisition phase, the foreperiod varied by group and was either constant at $2.7 \mathrm{~s}$ for the fixed group or sampled randomly from a continuous uniform distribution ranging between 1.7 and $3.7 \mathrm{~s}$ (mean $2.7 \mathrm{~s}$ ) for the random group. During the transfer phase, foreperiod distribution was identical for both groups and included $80 \%$ of the trials with foreperiod of $0.7 \mathrm{~s}$, and $20 \%$ of the trials with foreperiod of $2.7 \mathrm{~s}$. Instructions were provided before the first block. The accuracy rate of the previous block was presented during each break to facilitate task engagement.

\section{Eye tracking}

Binocular gaze position was monitored using a remote infrared video-oculographic system (Eyelink 1000 Plus; SR Research, Canada), with a spatial resolution $\leq 0.01^{\circ}$ and average accuracy of $0.25^{\circ}-0.5^{\circ}$ when using a headrest (as reported by the manufacturer). Raw gaze positions were sampled at $1000 \mathrm{~Hz}$ and converted into degrees of visual angle using a 9-point-grid calibration, which was performed at the beginning of the experimental session. Saccades were detected using a modification of a published algorithm ${ }^{63}$, which was applied on filtered gaze position data (low-pass IIR Butterworth filter; cutoff $60 \mathrm{~Hz}$; as in Amit et al., 2017). An elliptic threshold criterion for microsaccade detection was determined in $2 \mathrm{D}$ velocity space based on the horizontal and vertical velocities of eye-movement. We segmented the recorded eye movement data and created a segment of $2000 \mathrm{~ms}$ per trial starting from -1000 ms and ending at $+1000 \mathrm{~ms}$ relative to target onset at time zero. The median SD of the eye movements velocity was estimated based on these segments, and the microsaccade detection threshold was set to be six times the SD. Saccade onset was 
defined when six or more consecutive velocity samples were outside the ellipse, in both eyes. Saccades offsets are sometimes accompanied by an overshoot, which may be erroneously detected as a new saccade. Therefore, per standard procedure, we imposed a minimum criterion of $50 \mathrm{~ms}$ for the interval between two consecutive saccades and kept only the first saccade in cases where two saccades were detected within such interval. Saccades of all sizes were included in the analysis, but due to the instruction to keep sustained fixation, most saccades were small (in the range of microsaccades, $\left.<1^{\circ}\right)$.

The time series of saccade rate was constructed as in previous studies ${ }^{14,15,18,27,28}$ for each participant by counting the number of saccade events in each time-point across trials, separately for each group and block, and dividing these values by the number of trials (discounting trials in which a blink was detected in the given time sample).

\section{Statistical Analysis}

Behavioral analysis. Accuracy-rates and mean reaction time (RT) were calculated separately for each participant and group. Only correct trials were included in the RT analysis. Trials deviating by more than $3 \mathrm{SD}$ from the mean RT of each participant were also excluded from the data analysis. Analysis of the transfer phase included only the standard trials of foreperiod $0.7 \mathrm{~s}$ and not the rare trials of forperiod $2.7 \mathrm{~s}$.

Accuracy and RT were analyzed using generalized linear mixed models (GLMM) with fixed factors of Group (fixed/ random), Phase (acquisition /transfer), and the phase's trial ID (continuous). We constructed the GLMM assuming a binomial family of response and logit link (i.e., a logistic mixed effect model) for the 
accuracy model ${ }^{65}$ and a Gamma family of response with an identity link for the RT $\operatorname{model}^{66}$.

To capture the temporal dynamics across the phase, the trial ID was modeled using polynomials with increasing polynomial degrees fitted to the data. The polynomial models were iteratively compared using likelihood-ratio tests until there was no significant improvement in fit $(p>.05)$. Treatment contrasts were used for both Group and Phase factors, setting the fixed group and the acquisition phase as the base levels. Fixed effects were tested by comparing the effect against a reduced model without the effect (akin to type-II sum-of-squares, SS), using likelihood-ratio tests. Simple effects were examined using parameter coefficients and tested using the Wald z-test and the base levels were reset to examine some of the models' simple effects. To balance type-I and type-II errors ${ }^{67}$, the random effect structure of the model was chosen according to the model most parsimonious with the data ${ }^{68}$, as follows. First, a model allowing for a random intercept by subject was considered. Next, we tested whether a model with added random slopes by subject for fixed factors provided a better fit. Lastly, we tested whether a model with both random slopes by subject and random interaction slopes by subject better fits the data. In each iteration, we tested, using a likelihood-ratio test (against $a=.05$ ), whether the model converged, and, additional, whether there was a significant improvement in model fit relative to the reduced model. Models that failed to converge were trimmed from their random slopes or random interaction slopes, beginning with the random term that explains the least amount of variance, until reaching convergence.

Eye-movements analysis. In a series of previous studies, it was established that pre-target inhibition of saccade-rate reflects temporal expectation to a predicable target. Specifically, saccades were found to be inhibited shortly before predictable, 
but not unpredictable, targets ${ }^{14,15,18,26-28}$. Here, we used this index of pre-target saccade rate (SR) to examine the hypothesis that participants of the random group would show better adjustment, i.e., higher temporal expectation, to the new regularity during the transfer phase. To this end, we examined the mean pre-target SR: mean saccade rate at -100 to 0 ms relative to target onset, as was done in previous studies ${ }^{14}$. Pre-target SR was measured in the two groups during the transfer phase and compared between blocks. Another, subsidiary, goal of this analysis was to test whether the finding of enhanced SR inhibition for predictable, relative to unpredictable, targets holds even in a between-subject design. Since one group was presented with random foreperiods and the other with fixed foreperiods in the acquisition phase, this goal was achieved by comparing the pre-target SR of the two groups, in this phase.

Saccades occurrences are binary events - at each time point, a saccade either occurs or not. Therefore, SR can be measured on groups of trials and cannot be estimated in single trials, as was done for RT and accuracy-rates. In order to examine changes in SR across time, we calculated the mean pre-target SR (at -100 to $0 \mathrm{~ms}$ ) separately for each block of the transfer phase and compared between blocks. In addition, to test the second hypothesis, we compared the mean pre-target SR of both groups during the acquisition phase using an independent samples t-test.

Analysis software. GLMM analyses were done in R v4.0.3 using R-studio v1.3.959. We used the lme $4{ }^{69}$ package in $\mathrm{R}$, with model diagnostics done with the performance package $\mathrm{e}^{70}$. The behavioral outcomes and eye-movements results were processed in MATLAB R2020a (The MathWorks Inc., Natick, MA, USA). The statistical analyses were done in JASP v0.14 (JASP Team, 2020).

\section{Author Contributions.}


O. Shdeour and S. Yuval-Greenberg developed the study concept and design. Data collection, analysis and interpretation were performed by O. Shdeour. N. Tel-Perry helped with the statistical analysis. M. Glickman analyzed the results using the Drift Diffusion Model and contributed to its interpretation. The work was supervised by S. Yuval-Greenberg and funded by her research grants. All authors contributed to writing the manuscript and approved its final version for submission.

\section{Acknowledgement}

This study was funded by ISF grant $1960 / 19$ to S.Y-G and by Minducate award to O.S. 


\section{References}

1. Nobre, A. C., Correa, A., Coull, J. T., Mombaerts, P. \& Zador, T. The hazards of time. Curr. Opin. Neurobiol. 17, 465-470 (2007).

2. Nobre, A. C. \& Van Ede, F. Anticipated moments: Temporal structure in attention. Nat. Rev. Neurosci. 19, 34-48 (2018).

3. Fahle, M., Poggio, T. \& Poggio, T. A. Perceptual Learning. (MIT Press, 2002).

4. Goldstone, R. L. perceptual learning. Annu. Rev. Psychol. 49, 585-612 (2003).

5. Sagi, D. Perceptual learning in Vision Research. Vision Res. 51, 1552-1566 (2011).

6. Sasaki, Y., Nanez, J. E. \& Watanabe, T. Advances in visual perceptual learning and plasticity. Nat. Rev. Neurosci. 11, 53-60 (2009).

7. Sigman, M. \& Gilbert, C. D. Learning to find a shape. Nat. Neurosci. 3, 264 $269(2000)$.

8. Maniglia, M. \& Seitz, A. R. Towards a whole brain model of Perceptual Learning. Curr. Opin. Behav. Sci. 20, 47-55 (2018).

9. Wright, B. A. \& Zhang, Y. insights into Human Auditory Processing Gained from Perceptual Learning. in The cognitive neurosciences 353-367 (2009).

10. Wright, B. A., Buonomano, D. V., Mahncke, H. W. \& Merzenich, M. M. Learning and Generalization of Auditory Temporal-Interval Discrimination in Humans. J. Neurosci. 17, 3956-3963 (1997).

11. Bueti, D. \& Buonomano, D. V. Temporal perceptual learning. Timing Time Percept. 2, 261-289 (2014). 
12. Bartolo, R. \& Merchant, H. Learning and generalization of time production in humans: rules of transfer across modalities and interval durations. Exp. Brain Res. 197, 91-100 (2009).

13. Ying, X. An Overview of Overfitting and its Solutions. J. Phys. Conf. Ser. 1168, 022022 (2019).

14. Abeles, D., Amit, R., Tal-Perry, N., Carrasco, M. \& Yuval-Greenberg, S. Oculomotor inhibition precedes temporally expected auditory targets. Nat. Commun. 11, 3524 (2020).

15. Amit, R., Abeles, D., Carrasco, M. \& Yuval-Greenberg, S. Oculomotor inhibition reflects temporal expectations. Neuroimage 184, 279-292 (2019).

16. Baumeister, A. A. \& Joubert, C. E. Interactive effects on reaction time of preparatory interval length and preparatory interval frequency. J. Exp. Psychol. 82, 393 (1969).

17. Coull, J. T. \& Nobre, A. C. Where and when to pay attention: the neural systems for directing attention to spatial locations and to time intervals as revealed by both PET and fMRI. J. Neurosci. 18, 7426-7435 (1998).

18. Dankner, Y., Shalev, L., Carrasco, M. \& Yuval-Greenberg, S. Prestimulus Inhibition of Saccades in Adults With and Without Attention-Deficit / Hyperactivity Disorder as an Index of Temporal Expectations. Psychol. Sci. 28, 835-850 (2017).

19. Niemi, P. \& Näätänen, R. Foreperiod and simple reaction time. Psychol. Bull. (1981) doi:10.1037/0033-2909.89.1.133.

20. Näätänen, R. The diminishing time-uncertainty with the lapse of time after the 
warning signal in reaction-time experiments with varying fore-periods. Acta Psychol. (Amst). 34, 399-419 (1970).

21. Cravo, A. M., Rohenkohl, G., Wyart, V. \& Nobre, A. C. Temporal expectation enhances contrast sensitivity by phase entrainment of low-frequency oscillations in visual cortex. J. Neurosci. 33, 4002-4010 (2013).

22. Jepma, M., Wagenmakers, E. J. \& Nieuwenhuis, S. Temporal expectation and information processing: A model-based analysis. Cognition 122, 426-441 (2012).

23. Carrasco, M., Giordano, A. M. \& McElree, B. Temporal performance fields: visual and attentional factors. Vision Res. 44, 1351-1365 (2004).

24. Giordano, A. M., McElree, B. \& Carrasco, M. On the automaticity and flexibility of covert attention: A speed-accuracy trade-off analysis. J. Vis. 9, $30-30$ (2009).

25. Wickelgren, W. A. Speed-accuracy tradeoff and information processing dynamics. Acta Psychol. (Amst). 41, 67-85 (1977).

26. Badde, S., Myers, C. F., Yuval-Greenberg, S. \& Carrasco, M. Oculomotor freezing reflects tactile temporal expectation and aids tactile perception. Nat. Commun. 11, 1-9 (2020).

27. Tal-Perry, N. \& Yuval-Greenberg, S. Prestimulus inhibition of eye movements reflects temporal expectation rather than time estimation. Attention, Perception, Psychophys. 1-13 (2021) doi:10.3758/s13414-021-02319-9.

28. Tal-Perry, N. \& Yuval-Greenberg, S. Pre-target oculomotor inhibition reflects temporal orienting rather than certainty. Sci. Rep. 10, 1-9 (2020). 
29. Forstmann, B. U., Ratcliff, R. \& Wagenmakers, E. J. Sequential sampling models in cognitive neuroscience: Advantages, applications, and extensions. Annu. Rev. Psychol. 67, 641-666 (2016).

30. Ratcliff, R. \& G, M. The diffusion decision model: theory and data for twochoice decision tasks. Neural Comput. 20, 873-922 (2008).

31. Voss, A., Nagler, M. \& Lerche, V. Diffusion models in experimental psychology: A practical introduction. Exp. Psychol. 60, 385-402 (2013).

32. Glickman, M. \& Usher, M. Integration to boundary in decisions between numerical sequences. Cognition2019),193 ).

33. Glickman, M., Moran, R. \& Usher, M. Evidence integration and decisionconfidence are modulated by stimulus consistency. biorxiv.org2020) ).

34. Zhang, J. The Effects of Evidence Bounds on Decision-Making: Theoretical and Empirical Developments. Front. Psychol. 3, 263 (2012).

35. Voss, A. \& Voss, J. Fast-dm: A free program for efficient diffusion model analysis. Behav. Res. Methods 39, 767-775 (2007).

36. Schwarz, G. Estimating the dimension of a model. Ann. Stat. 461-464 (1978).

37. Cobbe, K., Klimov, O., Hesse, C., Kim, T. \& Schulman, J. Quantifying generalization in reinforcement learning. in International Conference on Machine Learning 1282-1289 (2019).

38. Nichol, A., Pfau, V., Hesse, C., Klimov, O. \& Openai, J. S. Gotta Learn Fast: A New Benchmark for Generalization in RL. (2018).

39. Reed, R. D. \& Marks, R. J. Neural smithing : supervised learning in 
feedforward artificial neural networks. (MIT Press, 1999).

40. Bishop, C. M. Neural networks for pattern recognition. (Clarendon Press, 1995).

41. Harris, H., Gliksberg, M. \& Sagi, D. Generalized Perceptual Learning in the Absence of Sensory Adaptation. Curr. Biol. 22, 1813-1817 (2012).

42. Wang, R., Zhang, J. Y., Klein, S. A., Levi, D. M. \& Yu, C. Task relevancy and demand modulate double-training enabled transfer of perceptual learning. Vision Res. 61, 33-38 (2012).

43. Xiao, L. Q. et al. Complete Transfer of Perceptual Learning across Retinal Locations Enabled by Double Training. Curr. Biol. 18, 1922-1926 (2008).

44. Szpiro, S. F. A., Wright, B. A. \& Carrasco, M. Learning one task by interleaving practice with another task. Vision Res. 101, 118-124 (2014).

45. Wright, B. A., Sabin, A. T., Zhang, Y., Marrone, N. \& Fitzgerald, M. B. Enhancing Perceptual Learning by Combining Practice with Periods of Additional Sensory Stimulation. J. Neurosci. 30, 12868-12877 (2010).

46. Brady, F. A Theoretical and Empirical Review of the Contextual Interference Effect and the Learning of Motor Skills. Quest 50, 266-293 (1998).

47. Magill, R. A. \& Hall, K. G. A review of the contextual interference effect in motor skill acquisition. Hum. Mov. Sci. 9, 241-289 (1990).

48. Albaret, J. M. \& Thon, B. Differential effects of task complexity on contextual interference in a drawing task. Acta Psychol. (Amst). 100, 9-24 (1998).

49. Cross, E. S., Schmitt, P. J. \& Grafton, S. T. Neural substrates of contextual 
interference during motor learning support a model of active preparation. $J$. Cogn. Neurosci. 19, 1854-1871 (2007).

50. de Lange, F. P., Heilbron, M. \& Kok, P. How Do Expectations Shape Perception? Trends Cogn. Sci. 22, 764-779 (2018).

51. Guo, K. et al. Effects on orientation perception of manipulating the spatiotemporal prior probability of stimuli. Vision Res. 44, 2349-2358 (2004).

52. Körding, K. P. \& Wolpert, D. M. Bayesian integration in sensorimotor learning. Nature 427, 244-247 (2004).

53. Geisler, W. S. \& Diehl, R. L. A Bayesian approach to the evolution of perceptual and cognitive systems. Cogn. Sci. 27, 379-402 (2003).

54. Knill, D. C. \& Richards, W. Perception as Bayesian Inference. (Cambridge University Press, 1996).

55. Yon, D. \& Frith, C. D. Precision and the Bayesian brain. Curr. Biol. 31, R1026-R1032 (2021).

56. Körding, K. P., Ku, S. \& Wolpert, D. M. Bayesian Integration in Force Estimation. J. Neurophysiol. 92, 3161-3165 (2004).

57. Miyazaki, M., Nozaki, D. \& Nakajima, Y. Testing Bayesian models of human coincidence timing. J. Neurophysiol. 94, 395-399 (2005).

58. Cicchini, G. M., Arrighi, R., Cecchetti, L., Giusti, M. \& Burr, D. C. Optimal encoding of interval timing in expert percussionists. J. Neurosci. 32, 10561060 (2012).

59. Jazayeri, M. \& Shadlen, M. N. Temporal context calibrates interval timing. 
Nat. Neurosci. 2010138 13, 1020-1026 (2010).

60. Rohenkohl, G., Cravo, A. M., Wyart, V. \& Nobre, A. C. Temporal Expectation Improves the Quality of Sensory Information. J. Neurosci. 32, 8424-8428 (2012).

61. Brainard, D. H. The Psychophysics Toolbox Short Title: The Psychophysics Toolbox Corresponding Author. Spat. Vis. 10, 433-436 (1997).

62. Menceloglu, M., Grabowecky, M. \& Suzuki, S. Comparing the effects of implicit and explicit temporal expectation on choice response time and response conflict. Attention, Perception, Psychophys. (2017) doi:10.3758/s13414-016-1230-4.

63. Engbert, R. Microsaccades: A microcosm for research on oculomotor control, attention, and visual perception. Prog. Brain Res. 154, 177-92 (2006).

64. Amit, R., Abeles, D., Bar-Gad, I. \& Yuval-Greenberg, S. Temporal dynamics of saccades explained by a self-paced process. Sci. Rep. 7, 1-15 (2017).

65. Jaeger, T. F. Categorical data analysis: Away fron ANOVAs (transformation or not) and towards Logit Mixed Models. J. Mem. Lang. 59, 434-446 (2008).

66. Lo, S. \& Andrews, S. To transform or not to transform: using generalized linear mixed models to analyse reaction time data. Front. Psychol. 6, 1-16 (2015).

67. Matuschek, H., Kliegl, R., Vasishth, S., Baayen, H. R. \& Bates, D. Balancing Type I error and power in linear mixed models. J. Mem. Lang. 94, 305-315 (2017).

68. Bates, D., Kliegl, R., Vasishth, S. \& Baayen, H. R. Parsimonious Mixed Models. arXiv (2015). 
69. Bates, D., Mächler, M., Bolker, B. \& Walker, S. Fitting Linear Mixed-Effects Models Using \{lme4\}. J. Stat. Softw. 67, 1-48 (2015).

70. Lüdecke, D., Makowski, D. \& Waggoner, P. performance: Assessment of Regression Models Performance. (2020). 